\title{
Mathematical Modeling of the Pandemic Peak
}

\author{
Nadezhda Cherkunova*(i) \\ Sayano-Shushensky Branch, Siberian Federal University, Republic of Khakassia, Russia
}

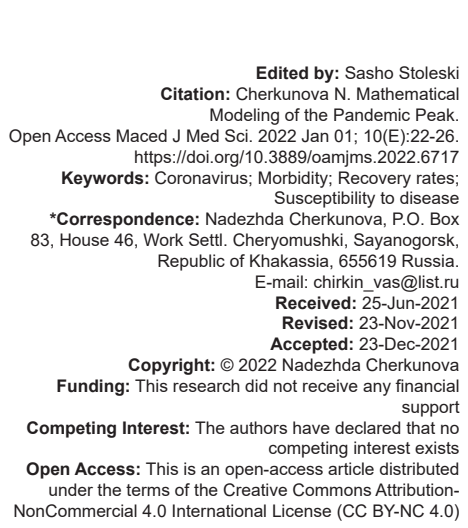

\section{Introduction}

For many centuries infectious diseases remain the most dangerous, which annually claim a large number of lives. Polio has accompanied humanity for thousands of years, paralyzing and killing thousands of children. To date, there is no effective drug against polio, although doctors are constantly improving the vaccine, which was developed in the early 1950s [1]. Humanity has been suffering from typhoid fever for centuries, and thousands of people have fallen victim to it. During the 30-year war in Europe (1618-1648), typhus, plague, and famine claimed the lives of 10 million people. During the First World War, the disease claimed several million lives in Russia, Poland, and Romania. The advent of the typhus vaccine during World War II helped to effectively eliminate the disease in the developed countries of the world. However, outbreaks still occur in some parts of South America, Africa, and Asia [2].

When the Europeans began to import African slaves to America, in addition to several new diseases, they brought also yellow fever. This disease destroyed entire cities. Despite vaccination and improved treatment methods, the epidemic still periodically breaks out in South America and Africa [3].
The emergence of AIDS in the 1980s led to a global pandemic, as more than 25 million people have died since 1981. According to the latest statistics, at present 33.2 million HIV $^{1}$-infected people live on the planet. At that, there is no cure for AIDS; however, some drugs can prevent HIV from transforming into AIDS [4].

During the $19^{\text {th }}$ century, traders non-deliberately exported the deadly cholera virus from India to cities in China, Japan, North Africa, the Middle East, and Europe. Over this time, six pandemics have killed millions of people. For decades, cholera seemed to be a disease of the past. However, a new strain of cholera emerged in 1961 in Indonesia and spread to most of the world [5].

Tuberculosis has ravaged the human population throughout history. Ancient texts tell in detail how the victims of the disease withered, while DNA testing revealed the presence of tuberculosis even in Egyptian mummies. Despite modern treatment methods, tuberculosis continues to affect eight million people annually, with deaths occurring in two million cases. The disease returned in the 1990s, mainly due to global poverty and the emergence of new, antibioticresistant strains of tuberculosis [6]. 
Specific figures on the impact of the first outbreaks of malaria are hard to come by. However, one can track the impact of malaria on humans by studying the regions affected by the disease. By the end of World War II, the World Health Organization (WHO) actively began to fight the disease around the world. The results obtained were not unambiguous; however, the cost of the project, the war, the emergence of a drugresistant new type of malaria and insecticide-resistant mosquitoes eventually led to the abandonment of the project. Today, malaria is still a problem in most countries of the world, especially in Africa [7].

The plague epidemic is considered the first plague pandemic, which killed half the population of Europe in 1348, and wiped out parts of the population of China and India. This disease has ruined many cities, constantly changed the structure of classes, and affected global politics, trade, and society. Today, the plague is still with humans. Today's medicine allows curing the disease at an early stage, therefore the risk of death is much lower [8].

When Europeans arrived in the Americas, they brought along many diseases to which the indigenous peoples had no immunity or protection. The main one among these diseases was smallpox. Despite the creation of a vaccine in 1796, the smallpox epidemic continued to spread. In 1967, the virus killed more than two million people, while millions of people around the world were severely affected by the disease [9].

The possibility of interspecific transmission of viruses depends on the intensity of contacts between different animals [10]. For example, the number of contacts between humans and bats is considered small: bats are not kept as pets and are not bred for food. Nevertheless, in some regions of the world, these animals are eaten. The diet of almost half of the inhabitants of villages in southern Cameroon includes bats [11]. Bats of this region are natural reservoirs of filoviruses that cause a dangerous disease, such as Ebola [12]. Ebola virus is a genus of viruses from the filovirus family that causes Ebola hemorrhagic fever in higher primates. The Ebola virus genus is divided into five species. Humans are affected by only four species. Zaire Ebola virus is considered the type species of the genus. It causes the largest number of outbreaks and has the highest mortality rate, reaching $90 \%$. There is still no specific treatment or vaccine for Ebola hemorrhagic fever.

The first reports of cases of a new coronavirus infection appeared in the city of Wuhan, Hubei Province (China) in December 2019 [13]. The infection spread rapidly throughout China, and on March 11, 2020, the WHO has reported that the COVID-2019 coronavirus outbreak has turned from an epidemic into a pandemic [14].

Coronaviruses are a large family of RNCcontaining viruses that cause zoonotic infections that are transmitted between animals and humans. At present, they are divided into four subfamilies and more than 30 species, whose list is constantly updated. The reason for the appearance of new coronaviruses that cause severe and rapidly spreading diseases is spontaneous mutations [15]. The entrance gate for infection is the mucosa of the upper respiratory tract. Further, virus reproduction occurs in the epithelium of the upper and lower respiratory tract, but to date, the pathogenetic mechanisms of the development of coronavirus infection have not been sufficiently studied.

At the initial stage of infection, the signs of coronavirus are practically the same as those of a common cold. If the disease is mild, lung inflammation does not develop, and the entire pathological process is limited to mild symptoms. In severe forms of the disease, the patient's condition quickly worsens, accompanied by high fervor and an unproductive persistent cough that results in respiratory failure. The patient's condition worsens significantly if there are concomitant diseases [16].

At present, the number of infected people in the world exceeds 60 million, while the number of deaths exceeds two million people. The search for a cure for the coronavirus is in full swing and is already yielding some results. To create community immunity, the population is vaccinated with vaccines that have passed three phases of clinical trials and have high effectiveness, low probability of side effects, and are recommended for mass use. While vaccines will help end the pandemic, they are unable to solve all the problems.

The globalization processes have led to the fact that several infections have become pandemics, affect the world community in general, and cause significant economic and social damage. Therefore, this problem has long outgrown state borders and has become a concern of the entire world community, but, despite the enormous efforts of humanity, it is still far from being resolved. Thus, the relevance of various studies on this problem is beyond doubt.

\section{Mathematical Modeling of the Pandemic Peak}

To study the spread of the pandemic, let draw up differential equations - a special case of the set of mathematical models that can be built when studying the world around [17].

Consider a sample of $\mathrm{n}$ people:

$$
n=x(t)+y(t)+z(t)
$$

where $x(t)$ is the number of infectious patients who are sick and simultaneously are the source of the spread of the disease at time $t$; 
$y(t)$ is the number of people susceptible to the disease, but healthy at time $t$;

$z(t)$ is the number of healthy people who are immune to this disease at time $t$.

Since the laws that make it possible to compose the equations of the process under consideration are unknown, it is therefore necessary to put forward various assumptions (hypotheses) concerning its course at small changes in variables.

Suppose that if $x(t)$ exceeds some threshold value of $X$, i.e. $x(t)>X$, then the rate of change in the number of people, susceptible to illness $\frac{d y}{d t}$ is proportional to their number $y(t)$,

$$
\frac{d y}{d t}=-a y
$$

Since each susceptible patient, who eventually becomes ill, becomes infectious himself, the rate of change in the number of infectious patients per unit of time $\frac{d x}{d t}$ represents the difference per unit of time between newly ill and those who are already recovering $\frac{d z}{d t}$

$$
\frac{d x}{d t}=a y-b x
$$

where the rate of change of convalescents is

$$
\frac{d z}{d t}=b x
$$

Where $a y, b x$ are the numbers of newly diseased and recovering.

$a$ and $b$ are the incidence and remission coefficients, respectively.

If $x(t) \leq X$, then $\frac{d y}{d t}=0$, and infection with the disease will not occur over time, since most infectious patients are isolated [18].

Let the incidence and remission coefficients be different, that is, $a \neq b$.

Assume that at the initial time $(\mathrm{t}=0)$ there are no patients with immunity to the disease.

$$
y(t)=y(0), x(t)=x(0), z(t)=z(0)=0
$$

Consider the case when $x(0)>X$. Since $x(t)$ is a continuous function, then there is an interval $[0 ; T)$, in which $x(t)>X$.

Separating the variables $\frac{d y}{y}=-a d t$ and integrating equation (1), we get $\ln |y|=-a t+c$ from where we find $y(t)=e^{c} \cdot e^{-a t}$.
Considering the initial conditions, we obtain the desired mathematical law describing the change in the number of people susceptible to the disease as a function of time:

$$
y(t)=y(0) \cdot e^{-a t}
$$

Substituting the found variable $y$ in equation (2), we obtain a linear differential equation of the $1^{\text {st }}$ order

$$
\frac{d x}{d t}+b x=a y(0) e^{-a t}
$$

Replacing $x(t)$ with the product of two auxiliary functions $(t)=u v$, we obtain a system of differential equations

$$
\left\{\begin{array}{c}
\frac{d v}{d t}+b v=0 \\
v \frac{d u}{d t}=a y(0) e^{-a t}
\end{array}\right.
$$

Dividing the variables in the $1^{\text {st }}$ equation of the system $\frac{d v}{v}=-b d t$ and integrating the resulting expression, we find the auxiliary function $v=e^{-b t}$.

Substituting the found value $v$ into the $2^{\text {nd }}$ equation of the system, separating the variables and integrating, we get the auxiliary function $u=\frac{a y(0)}{b-a} e^{(b-a) t}+C_{1}$

Considering the initial conditions (for $t=0$, $x(t)=x(0)$ ), we obtain the desired mathematical law describing changes in the number of susceptible to the disease depending on time

$$
x(t)=\frac{a y(0)}{b-a}\left(e^{(b-a) t}-e^{-b t}\right)+x(0) e^{-b t}
$$

The number of healthy people at a time $t$ is determined from the formula

$$
z(t)=n-x(t)-y(t)
$$

Let us find the peak of the pandemic, that is, the moment of time $T=t_{\max }$, at which the number of infectious patients will be the maximum.

Differentiating equation (4) we obtain the following equation

$$
\frac{a y(0)}{b-a}\left(b e^{-b t}-a e^{-a t}\right)-b x(0) e^{-b t}=0
$$

solving which we find the moment of time $\mathrm{T}=t_{\text {max }}$, at which the number of infectious patients will be the maximum, that is, the moment corresponding to the peak of the pandemic

$$
t_{\max }=\frac{\ln \left|\operatorname{aby}(0)-b^{2} x(0)+a b x(0)\right|-\ln ^{2} y(0)}{b-a}
$$




\section{Results}

The method of mathematical modeling based on differential equations (differential modeling) was used in the work. It was planned to find a time dependence of the number of people susceptible to the disease during pandemic, the number of infectious patients during pandemic, and the peak of the pandemic. For this purpose, differential equations were compiled for the rate of change in the number of susceptible to the disease $\frac{d y}{d t}$, infectious $\frac{d x}{d t}$, and recovering $\frac{d z}{d t}$ patients.

The case when the number of infectious patients $x(t)$ exceeds a certain threshold value $X, x(t)>X$ has been considered.

For various coefficients of morbidity $a$ and recovery $b$, and assuming the absence of patients with immunity at the initial time $t=0$, the desired epidemiological parameters were obtained using solutions to differential equations of the constructed model. These parameters reflect laws of change in the number of susceptible to the disease depending on time

$$
y(t)=y(0) \cdot e^{-a t}
$$

and the time-dependent number of infectious patients

$$
x(t)=\frac{a y(0)}{b-a}\left(e^{-a t}-e^{-b t}\right)+x(0) e^{-b t}
$$

within the interval $(0 ; \mathrm{T})$.

If $x(t) \leq X$, then $\frac{d y}{d t}=0$ and infection with the

disease will not occur over time, since most infectious patients are in isolation.

Using the equation for $\mathrm{x}(\mathrm{t})$, the moment $\mathrm{T}=t_{\max }$ was found, at which the number of infectious patients will be the maximum, that is, the moment corresponding to the peak of the pandemic

$$
t_{\max }=\frac{\ln \left|a b y(0)-b^{2} x(0)+a b x(0)\right|-\ln ^{2} y(0)}{b-a}
$$

\section{Discussion}

Mathematical modeling is indispensable in certain areas of medicine where real experiments are impossible or difficult, for example, in epidemiology. The most well-known and paradigmatic model in mathematical epidemiology is the simple SIR (Standardized Infection Ratio) model, described by Kermack and McKendrick. This model describes the dynamics of groups of susceptible, infected, and recovered individuals using systems of differential equations (continuous-time) or difference equations (discrete-time) [19]. Works of Bartlett's research on the stochastic SIR model in continuous time gave rise to the development of stochastic models of epidemiological processes [20]. Besides, the work of Baily [21] made a great contribution to the development of applications of the theory of random processes to the modeling of epidemics. One of the first spatial models of epidemic spread, based on partial differential equations, was described by Kendall [22].

Since then, significant progress has been made in the construction of stochastic models, but the creation of real mathematical epidemiology is just beginning. In the mathematical theory of epidemics, even more sharply than in the study of populations, a serious contradiction is revealed between the requirement of the realism of the model and the possibility of its analysis. Even the simplest stochastic models of the spread of epidemics are extremely complex from a mathematical standpoint. Therefore, the issue of which model to choose for the study and which methods of analysis to use are crucial. The main principle of mathematical modeling of complex systems is the principle of optimality [23]. This means that the model should be as simple as possible, that is, it should contain a minimum number of variables (and, consequently, equations) and also have relatively simple relationships between variables.

Models, based on differential equations occupy a special place among the existing models of the spread of infectious diseases. They may be less realistic than stochastic models, but they are more convenient for analysis.

The analysis of existing epidemic models [24] indicates that all of them are based on the division of the population into groups. The epidemic situation is described by a system of equations (usually differential), which, depending on the type of model, in one way or another determines the dynamics of the transition of individuals from one group to another. Differential models make it possible not only to predict the development of the situation but also to evaluate various epidemiological parameters.

\section{References}

1. Murray D. Infectious Diseases in Children. Moscow: Praktika 2006.

2. Timakov VD, Levashev VS, Borisov LB. Microbiology. Moscow: Medicine; 1983.

3. Smorodintsev AA. Conversations about Viruses. Moscow Eureka; 1982.

4. Khaitov RM, Ignatieva GA. Narodnaya Akademiya Kul'tury Obshchechelovecheskih Cennostej. Moscow: People's 
Academy of Culture and Universal Values; 1992.

5. Zlatogorov SI. Epidemiology and Microbiology of Cholera. Moscow; Oxford University Press; 1947.

6. Samtsov AV, Barbinov VV. Skin and Venereal Diseases. Moscow: ELBI; 2002.

7. Greenwood BM, Bojang K, Whitty CJ, Targett GA. Malaria Lancet. 2005;365(9469):1487-98. https://doi.org/10.1016/ S0140-6736(05)66420-3 PMid:15850634

8. Domaradsky IV. The Plague. Moscow: Medicine; 1998.

9. Kazantsev AP, Matkovsky VS. Handbook of Infectious Diseases. Moscow: Medicine; 1985.

10. Parrish CR, Holmes EC, Morens DM, Park EC, Burke DS Calisher $\mathrm{CH}$, et al. Cross-species virus transmission and the emergence of new epidemic diseases. Microbiol Mol Biol Rev. 2008;72(3):457-70. https://doi.org/10.1128/MMBR.00004-08 PMid:18772285

11. Baudel $H$, De Nys $H$, Ngole EM, Peeters $M$, Desclaux A. Understanding Ebola virus and other zoonotic transmission risks through human-bat contacts: Exploratory study on knowledge, attitudes, and practices in Southern Cameroon. Zoonoses Public Health. 2019;66(3):288-95. https://doi. org/10.1111/zph.12563 PMid:30677236

12. Leroy $E M$, Kumulungui $B$, Pourrut $X$, Rouquet $P$, Hassanin $A$. Fruit bats as reservoirs of Ebola virus. Nature. 2005;438:575-6. https://doi.org/10.1038/438575a

PMid: 16319873

13. Dong $\mathrm{Y}$, Mo $\mathrm{X}$, Hu $\mathrm{Y}$, Qi $\mathrm{X}$, Jiang $\mathrm{F}$, Jiang $\mathrm{Z}$, et al. Epidemiology of COVID-19 among children in China.
Pediatrics. 2020;145(6):e20200702. https://doi.org/10.1542/ peds.2020-0702

PMid:32179660

14. Coronavirus Disease (COVID-19) Pandemic. Geneva: World Health Organization; 2020. Available from: https://www.who.int/ emergencies/diseases/novel-coronavirus-2019 [Last accessed on 2021 Oct 20].

15. Paules $\mathrm{Cl}$, Marston HD, Fauci AS. Coronavirus Infections-more Than Just the Common Cold. JAMA. 2020;323(8):707.

16. Coronavirus: Symptoms and Prophylactics; 2021. Available from: https://www.medikom.ua/ru/koronavirus-simptomy-iprofilaktika [Last accessed on 2021 Oct 20].

17. Cherkunova NG. Mathematical modeling of economic processes. Espac Bus Int Manag. 2017;38(48):7.

18. Braun M. Differential Equation Models. New York: Springer; 1983

19. Kermack WO, McKendrickAG. Acontribution to the mathematical theory of epidemics. Proc R Soc 1927;115(772):700-21.

20. Bartlett MS. An Introduction to Stochastic Processes, with Special Reference to Methods and Applications. $3^{\text {rd }}$ ed. Cambridge: Cambridge University Press; 1978. p. 388.

21. Bailey N. Mathematics in biology and medicine. Moscow: Mir; 1970. p. 327.

22. Kendall DG. Discussion of 'Measles periodicity and community size. J Roy Stat Soc Ser A. 1957;120:64-76.

23. Balanter $\mathrm{BI}$, Khanin MA, Chernavsky DS. Introduction to Mathematical Modeling of Pathological Processes. Moscow: Medicine; 1980. p. 262.

24. Gray A, Greenhalgh D, Mao X, Pan J. The SIS Epidemic Model with Markovian Switching. Available from: http://www. strathprints.strath.ac.uk/41322 [Last accessed on 2021 Oct 20]. 